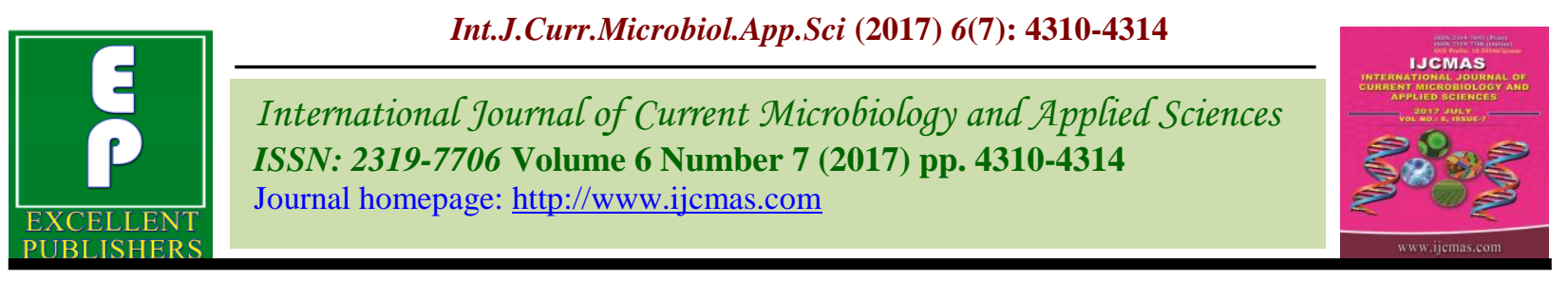

Original Research Article https://doi.org/10.20546/ijcmas.2017.607.448

\title{
Diagnostic Value of Sputum Gram's Stain and Sputum Culture in Lower Respiratory Tract Infections in a Tertiary Care Hospital
}

\author{
Akansha Rana ${ }^{1 *}$, Archana Sharma ${ }^{1}$ and Gaganpriya Pandey ${ }^{2}$ \\ ${ }^{1}$ Department of Microbiology, WCMS\&R, Jhajjar, Haryana, India \\ ${ }^{2}$ Department of Microbiology, NIMS (Paramedical College), Jaipur, Rajasthan, India \\ *Corresponding author
}

\section{A B S T R A C T}

\section{Keywords}

Gram's stain, Sputum culture, Lower respiratory tract infections, Bartlett's criteria, Streptococcus pneumoniae, Klebsiella pneumoniae.

Article Info

Accepted:

29 June 2017

Available Online:

10 July 2017
There has always been a controversy in bacteriological assessment of sputum samples in diagnosing lower respiratory tract infections (LRTIs). Commonly in the Microbiological laboratory, expectorated sputum samples are microscopically examined for diagnosing LRTIs. The study was aimed to determine the diagnostic value of sputum gram's stain and sputum culture in Lower Respiratory Tract Infections in a tertiary care hospital. Lower respiratory tract secretion (sputum) of 233 patients was cultured, identified and antimicrobial susceptibility by Kirby-Bauer disc-diffusion method was performed by standard methods. Quality of expectorated sputum samples were assessed by using Bartlett's grading system. Among acceptable category, 141(77.05\%) samples showed culture positivity. Among non-acceptable category, 42(22.92\%) samples showed culture positivity. Streptococcus pneumoniae $37(20.22 \%$ ) was the commonest isolated organism followed by Klebsiella pnemoniae-30(16.39\%), Escherichia coli-22(12.03\%), Staphylococcus aureus-21(11.47\%) and Pseudomonas aeruginosa-18(09.84\%). In this study, authors have recommended receiving good quality of sputum and the subsequent Gram stain and culture of sputum can provide a high diagnostic yield for clinically relevant LRTIs.

\section{Introduction}

Lower respiratory tract infections (LRTIs) are the commonest health problem demanding frequent consultation and hospitalization. LRTIs are among the most common infectious diseases of humans' worldwide (Carroll, 2002). Commonly used method in the laboratory for diagnosing LRTI is the microscopic examination of sputum and its culture. The lower respiratory tract secretion (sputum) is usually contaminated with normal flora of the oropharynx or saliva (upper respiratory tract secretions). So, a large number of different species overgrow in sputum culture thus preventing the determination of the true pathogen (Nihan Ziyade and Aysegul Yagci, 2010). Sputum sample sent to the laboratory should be deepcoughed and purulent instead of watery saliva sputum, which leads to erroneous results. Thus, collection of sputum sample, sputum microscopy and culture is very important for the diagnosis and management of LRTIs. The usefulness of Gram staining of sputum samples in the initial approach to a patient with LRTIs is still controversial. Data from previous studies that vouch for its utility have 
also shown a limited sensitivity, but data also have shown a specificity of $>80 \%$ for the diagnosis of pneumococcal pneumonia (Gleckman et al., 1988; Bohte et al., 1996 and Schmid et al., 1979). However, some authorities feel that there is no strong evidence in favour of its everyday use in diagnosing community acquired pneumonia (CAP). Indeed, although the Infectious Diseases Society of America guidelines recommend Gram staining of expectorated sputum for patients requiring hospitalization, while the American Thoracic Society does not (Barlett et al., 1998 and the ATS board of directors, guidelines, 1993). Hence, the present study was undertaken to analyse the importance of sputum's gram's stain and sputum culture in patients with Lower Respiratory Tract Infections.

\section{Materials and Methods}

The present study was carried out in the time span of 6 months from Jan 2015 to June 2015 in the Department of Microbiology NIMS Medical College, Jaipur. A total of 233 sputum samples were received in diagnostic microbiology for culture and sensitivity. All specimens belonged to patients suspected of having LRTIs. Purulent portion of samples were used for making smears for Gram staining and for inoculating culture media. The specimens were cultured on Blood agar, MacConkey agar and Chocolate agar and incubated at $37^{\circ} \mathrm{C}$ for $18-24$ hours. Identification of bacterial isolates was done by their characteristic appearance on the media, Gram's staining, motility testing (by hanging drop method), and biochemical tests (Catalase, Coagulase, Indole, Methyl red, voges-proskauer, Citrate, Urease, Triple sugar iron, PPA, Oxidase test). Antimicrobial susceptibility testing by Modified Kirby Bauer's disc diffusion method according to the clinical laboratory standard institute (CLSI guidelines, 2014) was done. Results of Gram stained smears were interpreted based on the presence of microorganisms, pus cells and epithelial cells, seen under microscope

Quality of expectorated sputum samples was assessed by using Bartlett's grading system and a score was given below.

The pus cells and epithelial cells were observed under microscope in 20-30 low power fields (LPF) and average number of epithelial cells and pus cells were calculated and thus the total score is derived.

The final score value of less than or equal to zero indicates a salivary contamination of sputum sample (non- acceptable sputum sample). The final score of 1 and above was considered to accept sputum sample.

\section{Results and Discussion}

Based on Bartlett's screening criteria, out of 233 sputum samples processed, 167 (71.62\%) were acceptable and 66 (28.33\%) were non-acceptable (Table 1). Potential pathogens were obtained from 183 of 233 samples, of which 141 are from acceptable samples $(77.05 \%)$, and 42 are from nonacceptable samples $(22.92 \%)$ (Table 3$)$.

Following pathogens were isolated from sputum culture in patients having clinically suspected LRTIs (Table 2).

The occurrence of bacterial pathogens varies with age, 41-60years $(41.21 \%)$ recorded higher isolates while age group 1-20years recorded the least $(12.88 \%)$. Sex related occurrence of pathogens reveals that, male $171(73.4 \%)$ subjects reported higher number of pathogens compared to females $62(26.6 \%)$. The organisms obtained from the nonacceptable category (42 of 66) included, Pseudomonas aeruginosa- 6, Staphylococcus aureus-8, Klebsiella pneumoniae- 15, Escherichia coli- 11 and Citrobacter spp.- 2. 
Sputum samples are commonly examined in the Microbiological laboratory to diagnose LRTIs. However, sputum will be contaminated with upper respiratory tract secretions, i.e., saliva. For this reason, sputum is among the least clinically relevant specimen received for culture in microbiology laboratories, even though it is one of the most common and timeconsuming specimen. Good sputum samples depend on thorough healthcare worker education and patient understanding throughout all phases of the collection process (Fuselier et al., 2002). Bartlett's sputum grading system is not applicable for lower respiratory tract infections caused by viruses, fungi, Mycobacterium tuberculosis and Legionella species. The importance of micro-organisms recovered from respiratory samples must always be evaluated in light of clinical history (Washington Winn et al., 2006).

In Present study 233 sputum samples were processed, $167(71.62 \%)$ were acceptable and $66(28.33 \%)$ were non-acceptable based on Bartlett's screening criteria, which is similar with the study conducted by Anevlavis et al., (2009) and Mariraj et al., (2011) who had reported their acceptability percentages as $63 \%$ and $79 \%$, respectively. In contrast, Daniel Musher et al., (2004) had reported a low percentage of $31 \%$ acceptability.

Table.1 Bartlett's Criteria[6] used

\begin{tabular}{|l|l|}
\hline Number of Neutrophils /10X LPF & GRADE \\
\hline$<10$ & 0 \\
\hline $10-25$ & +1 \\
\hline$>25$ & +2 \\
\hline Presence of mucus & +1 \\
\hline Number of Epithelial Cells /10X LPF \\
\hline $10-25$ & -1 \\
\hline$>25$ & -2 \\
\hline TOTAL SCORE & \\
\hline
\end{tabular}

Table.2 Distribution of micro-organisms isolated from sputum

\begin{tabular}{|l|l|l|}
\hline Isolates & \multicolumn{1}{|c|}{ Sputum $\mathrm{n}=183$} & $(\%)$ \\
\hline Streptococcus pneumonia & 37 & 20.22 \\
\hline Streptococcus pyogenes & 27 & 14.76 \\
\hline Klebsiella pneumonia & 30 & 16.39 \\
\hline Pseudomonas aeruginosa & 18 & 09.84 \\
\hline Staphylococcus aureus & 21 & 11.47 \\
\hline Escherichia coli & 22 & 12.03 \\
\hline Citrobacter spp. & 07 & 03.83 \\
\hline Enterococcus spp. & 08 & 04.37 \\
\hline Enterobacter spp. & 03 & 01.63 \\
\hline Acinetobacter spp. & 02 & 01.09 \\
\hline Candida spp. & 08 & 04.38 \\
\hline
\end{tabular}


Table.3 Gram smear and culture result of sputum samples

\begin{tabular}{|l|l|l|l|}
\hline $\begin{array}{l}\text { Gram } \\
\text { smear }\end{array}$ & $\begin{array}{l}\text { Culture } \\
\text { positive }\end{array}$ & $\begin{array}{l}\text { Culture } \\
\text { negative }\end{array}$ & Total \\
\hline Positive & 141 & 26 & 167 \\
\hline Negative & 42 & 24 & 66 \\
\hline Total & 183 & 50 & 233 \\
\hline
\end{tabular}

Parry et al., (2000) suggested that sputum Gram smear can be a guide to the etiology of pneumonia, particularly pneumococcal pneumonia. Whereas Ewig et al., (2002) did not recommend sputum collection for diagnosis of community acquired pneumonia and suggested that Gram stain had a low diagnostic yield and a low number of positive samples had a corresponding growth in culture.

Total culture positivity in the present study was $78.54 \%$ (183/233). Culture positivity reported in other studies include- Jean Lloveras et al., (2010) - 57\% and Daniel Musher et al., (2004) - 79\%. On the contrary Ravichandran et al., (2001) had reported 5\% of culture positivity.

It has been suggested that the value of Gram stain and culture results are dependent upon the pretest probability that the patient has bacterial pneumonia and upon whether the patient has received antibiotics (Carroll, 2002).

The most common pathogen causing lower respiratory tract infection isolated was Streptococcus pneumoniae 37(20.22\%) followed by Klebsiella pneumoniae 30(16.39\%), Streptococcus pyogens 27(14.76\%), Escherichia coli 22(12.03\%) and Pseudomonas aeruginosa 18(09.84\%). Other organisms are represented in table 2.

A useful tool in the quality assurance of sputum culture is the comparison of primary gram's stain and culture of the sputum
(Chinnnusamy et al., 2016). Report of gram staining of sputum reflects microbial flora of lower respiratory tract provided the sputum is of good quality. Role of pathogens which are isolated from non acceptable sputum samples causing LRTIs is uncertain.

In conclusion, when the pathogens are identified accurately management of LRTI is remarkably simplified. Value of sputum Gram stain and culture is controversial for diagnosis in LRTIs. In this study, the authors recommended to receive a good quality of sputum and subsequent Gram staining of sputum and sputum culture can provide a high diagnostic yield for clinically relevant LRTIs.

\section{References}

Barlett, J.G., Breiman, R.F., Mandell, L.A., File, T.M. 1998. Community-acquired pneumonia in adults: guidelines for management. Clin. Infect. Dis., 26: 81138 .

Bohte, R., Hermans, J., van den Broek, P.J. 1996. Early recognition of Streptococcus pneumoniae in patients with communityacquired pneumonia. Eur. J. Clin. Microbiol. Infect. Dis., 15: 201-5.

Carroll, K.C. 2002. Laboratory diagnosis of lower respiratory tract infections: controversy and conundrums. J. Clin. Microbiol., 40: 3115-3120.

Chinnnusamy, N., Arumugam, V., Vedachalam, D. 2016. A Study on Analysis of the Sputum Gram Staining and Culture in Patients with Lower Respiratory Tract Infections Attending a Tertiary Care Hospital. Indian J. Microbiol. Res., 3(1): 
24-26.

CLSI (Clinical and laboratory standards institute) guidelines, 2014. $\mathrm{M}_{02}-\mathrm{A}_{11}, \mathrm{M}_{07^{-}}$ $\mathrm{A}^{9}$, and $\mathrm{M}^{11}-\mathrm{A}^{8}$.

Daniel, M., Musher, Roberto Montoya, Anna Wanahita. 2004. Diagnostic value of microscopic examination of Gram stained sputum and sputum cultures in patients with Pneumococcal pneumonia. Clin. Infect. Dis., 39: 165-169.

Ewig, S., Schlochtermeter, M., Göke, N., Niederman, M.S. 2002. Applying sputum as a diagnostic tool in pneumonia. Limited yield, minimal impact on treatment decisions. Chest, 121:1486-1492.

Fuselier, P.A., Garcin, L.S., Procop, G.W. 2002. Infections of the Lower Respiratory Tract. In: Betty AF, Daniel FS, Alice SW, editors. Bai ley and Scott's Diagnostic Microbiology. Mosby, 884-898.

Gleckman, R., DeVita, J., Hibert, D., Pelleiter, C., Martin, R. 1988. Sputum Gram stain assessment in community-acquired bacteremic pneumonia. J. Clin. Microbiol., 26: 846-9.

Jean Jacques Lloveras, Mohamed -Issam, Shukr, Claude Pinos, Anissa Lindoulsi, Philippe Grima. 2010. Usefulness of sputum Gram'sstain and culture for diagnosis of pneumonia in Geriatric institution. J. IMAB, 16(3): 20 - 22.

Mariraj, J., Surekha, Y., Asangi, Krishna, S., Suresh, B., Sonth, Ramesh, Shanmugam. 2011. Sputum Gram's stain assessment in relation to sputum culture for Respiratory Tract Infections in a tertiary care hospital. J. Clin. Diag. Res., 5(8): 1699-1700.

Nihan Ziyade, Aysegul Yagci. Improving sputum culture results for diagnosis of lower respiratory tract infections by saline washing. Marmara Med. J., 23(1): 30-36.

Parry, C.M., White, R.R., Ridgeway, E.R., Corkill, J.E., Smith, G.W. 2000. The reproducibility of sputum Gram film interpretation. J. Infect., 41: 55-60.

Ravichandran Theerthakarai, Walid El Halees, Medhat Ismail, Roberto, A., Solis, M., Anees Khan. 2001. Non value of Initial Microbiological Studies in the management of non-severe Community Acquired Pneumonia. Chest, 119(1): 181184.

Schmid, R.E., Anhalt, J.P., Wold, A.D., Keys, T.F., Washington, J.A. 1979. Sputum counter- immuno-electrophoresis in the diagnosis of pneumococcal pneumonia. Am. Rev. Respir. Dis., 119: 345-8.

Stavros Anevlavis, Niki Petroglon, Athanasios Tzavaras, Efstratios Maltzos, Ioannis Pneumatikos, Marios Froudarakis, Eleftherios Anevlavis, Demosthenes Bouros. 2009. A prospective study of the diagnostic utility of sputum Gram stain in pneumonia. J. Infect., 59: 83-89.

The ATS Board of Directors. 1993. Guidelines for the initial management of adults with community-acquired pneumonia: diagnosis, assessment of severity, and initial antimicrobial therapy. Am. Rev. Respir. Dis., 148: 1418-26.

Washington Winn Jr, Stephen Allen, William Janda, Elmer Koneman. 2006. Guidelines for collection, transport, processing, analysis and reporting of cultures from specific specimen sources. In: Koneman's colour atlas and textbook of Microbiology, 6th edition. Lippincott, Williams and Wilkins publications, 68-111.

\section{How to cite this article:}

Akansha Rana, Archana Sharma and Gaganpriya Pandey. 2017. Diagnostic Value of Sputum Gram's Stain and Sputum Culture in Lower Respiratory Tract Infections in a Tertiary Care Hospital. Int.J.Curr.Microbiol.App.Sci. 6(7): 4310-4314.

doi: https://doi.org/10.20546/ijcmas.2017.607.448 